\title{
Sheltering efficiency of wind barriers on bridges
}

ARTICLE in JOURNAL OF WIND ENGINEERING AND INDUSTRIAL AERODYNAMICS • AUGUST 2012

Impact Factor: $1.41 \cdot$ DOI: 10.1016/j.jweia.2012.04.027

CITATIONS

15

4 AUTHORS, INCLUDING:

Hrvoje Kozmar

University of Zagreb

37 PUBLICATIONS 173 CITATIONS

SEE PROFILE

\section{Gianni Bartoli}

University of Florence

109 PUBLICATIONS 537 CITATIONS

SEE PROFILE
READS
Lorenzo Procino

University of Florence

23 PUBLICATIONS 59 CITATIONS

SEE PROFILE 


\title{
Sheltering efficiency of wind barriers on bridges
}

\author{
Hrvoje Kozmar $^{\mathrm{a}, *}$, Lorenzo Procino ${ }^{\mathrm{b}}$, Alessandra Borsani ${ }^{\mathrm{b}}$, Gianni Bartoli ${ }^{\mathrm{b}}$ \\ ${ }^{a}$ Faculty of Mechanical Engineering and Naval Architecture, University of Zagreb, Ivana Lučića 5, 10000 Zagreb, Croatia \\ ${ }^{\mathrm{b}}$ Department of Civil and Environmental Engineering, University of Florence, Via di Santa Marta 3, 50139 Florence, Italy
}

\section{A R T I C L E I N F O}

\section{Article history:}

Received 20 July 2011

Received in revised form

22 April 2012

Accepted 30 April 2012

Available online 2 June 2012

Keywords:

Sheltering efficiency of wind barriers Bridges

Airflow field characteristics

Wind-tunnel tests

\begin{abstract}
A B S T R A C T
Sheltering efficiency of wind barriers on viaducts was experimentally studied in a boundary layer wind tunnel. Effects of wind incidence angle on flow field characteristics in the wake of a wind barrier were reported. Mean velocity fields and vorticity fields were determined using the Particle Image Velocimetry (PIV) technique. Freestream velocities were measured using hot-wire and Pitot tube. Results indicate a possibility of wind-induced instability of high-sided vehicles at larger vertical incidence angles, especially in the traffic lane close to trailing edge of the bridge, as velocity fluctuations and mean freestream velocities approach the road surface when increasing the vertical incidence angle. Removing elements from the wind barrier causes very large local velocities immediately downstream from a barrier and strong vorticity in the entire area in the wake of a wind barrier. Variations in horizontal incidence angle do not seem to affect flow field characteristics significantly. Without a wind barrier, wind velocities on bridges reach $80 \%$ of the freestream velocity at height as low as $1 \mathrm{~m}$ full-scale along with very strong vorticity in the immediate vicinity of the road surface.
\end{abstract}

(c) 2012 Elsevier Ltd. All rights reserved.

\section{Introduction}

Strong bora winds on both the Italian and the Croatian coast induce instability of vehicles, especially on viaducts and bridges, and in the past freeways have had to be closed for traffic often due to safety requirements. Recently, an extensive research program has been undertaken to develop an optimal design of wind barriers for several freeway viaducts particularly exposed to cross-winds.

Some of the first studies on aerodynamic design of wind barriers in modern times are reported by Nägeli (1941) and Jensen (1954), whereas most of the research was carried out for agricultural purposes. Kaiser (1959) was among the first researchers who made a distinction between 'mean wind reduction' and 'wind protection', emphasizing that while a less porous wind barrier may give a greater reduction in mean wind velocity, the greater turbulence in its wake may reduce its overall effectiveness for wind protection compared to a more porous wind barrier. Both Jensen (1958) and Kaiser (1959) indicated that flow characteristics in the wake of wind barriers are independent of approaching flow velocity for neutrally-stratified aerodynamically rough flow. Moreover, Raine (1974) and Raine and Stevenson (1977) pointed out that Reynolds number similarity is relatively unimportant for wind barrier aerodynamics, while turbulence in oncoming flow is

\footnotetext{
*Corresponding author. Tel.: +38516168 162; fax: +38516156940.

E-mail addresses: hrvoje.kozmar@fsb.hr (H. Kozmar), lorenzo.procino@unifi.it (L. Procino), alessandra.borsani@pin.unifi.it (A. Borsani), gianni.bartoli@unifi.it (G. Bartoli).
}

very important. Arie and Rouse (1956), Good and Joubert (1968) and de Bray (1971) indicated that an increased roughness of the upstream terrain (more turbulent flow) reduces the wind-barrier drag coefficient and reattachment distance due to a larger vertical exchange of streamwise momentum resulting from higher Reynolds stress $u^{\prime} w^{\prime}$, which intensifies a recovery of streamwise momentum behind a wind barrier $\left(u^{\prime}\right.$ and $w^{\prime}$ are fluctuating velocity components in the main wind direction and vertical direction, respectively). McNaughton (1988) reported a quiet zone of reduced turbulence and smaller eddy size immediately behind wind barriers independent of the barrier porosity. Further downwind an extended wake region of increased turbulence with eddy sizes recovering to upwind length scales was observed.

Wind barriers generally reduce turbulent eddy length, thus increasing the peak frequency of turbulent velocity fluctuations (Heisler and DeWalle, 1988), while peak frequency of velocity fluctuations close to wind barriers tends to increase with barrier wall porosity. Several studies reported that for some windbreak configurations the wind-protected area is larger for consecutively arranged windbreaks compared to a single windbreak (e.g. McAneney and Judd, 1991; Judd et al., 1996; Dierickx et al., 2001; Frank and Ruck, 2005). Wind-tunnel experiments by Cornelis and Gabriels (2005) indicated that porosity from 20\% to 35\% (ratio between open area and entire barrier wall) could be optimal in terms of wind velocity reduction, while Jensen (1954) and Blenk and Trienes (1956) showed a maximum sheltering efficiency to be associated with porosities from $35 \%$ to $50 \%$. Dong et al. (2007) indicated that the optimal porosity could be between $20 \%$ and $30 \%$ 
suggesting that for porosity higher than $30 \%$ the bleed flow dominates and for porosity lower than 20\% the reversed flow becomes significant. As the discrepancy between these results is quite large, it seems that the sheltering efficiency strongly depends even on small details of wind barrier design and oncoming wind turbulence. Cornelis and Gabriels (2005) reported that an evenly distributed porosity in the barrier wall generated the longest protected area, noting that the optimal design of wind barrier wall depends strongly on their purpose. Dierickx et al. (2003) showed that windbreaks are less effective for rough than for smooth turbulent flow, although differences depend on the open area of the windbreak. Moreover, they indicated that inclination of wind screens may influence their efficiency, in agreement with Nord (1991). Plate (1971) showed that separation from the top of the shelterbelt gives rise to a separation streamline which divides the low velocity flow below from the high velocity flow aloft. The blending of the flow across this streamline, which determines the recovery of the wind profile and the reduction in sheltering efficiency, is caused by the gradient in velocity across the streamline, while its location is determined by the drag on the shelter and the pressure distribution behind it.

Several researchers attempted to quantify aerodynamic characteristics of wind barriers in a form of a sheltering coefficient. Miller et al. (1975) suggested a shelterbelt drag, characterized by the integrated wind reduction curve or a drag coefficient, as a practical basis for comparison of the effectiveness of different field shelterbelts. Gandemer $(1979,1981)$ suggested a shelter parameter based on the generally accepted critical level of discomfort, and the corresponding discomfort wind frequencies. Schwartz et al. (1995) developed an equation to describe the near ground horizontal distribution of mean relative velocity in the vicinity of the barrier.

Even though the above mentioned studies provide valuable information on aerodynamic features of wind barriers placed on ground surface, their results are not sufficient to properly design wind barriers on bridges, as the aerodynamics of agricultural wind barriers immersed in the atmospheric boundary layer differs significantly from aerodynamics of wind barriers on bridges. Moreover, very different designs of bridges resulting in significantly different flow features require the aerodynamics of wind barriers to be investigated for each object as a separate study. Previously, Štrukelj et al. (2005) numerically studied effects of wind barrier geometry on wind forces experienced by vehicles on the Črni Kal viaduct in Slovenia. Wang et al. (2007) designed a wind barrier to reduce wind velocities on the bridge deck of the Hangzhou Bay Bridge in China based on wind-tunnel experiments and numerical simulations using the Random Vortex Method (RVM). Results reported in Procino et al. (2008) indicate a decrease in velocities on the bridge with reduced barrier porosity and increased barrier wall height. Flow field characteristics on the Bukovo viaduct proved to be significantly improved using a $4 \mathrm{~m}$ high wind barrier compared to the configuration without a wind barrier, Kozmar et al. (2009a).

In this paper, effects of wind incidence angle on flow field characteristics in the wake of wind barriers are reported for two viaducts on the A6 Rijeka-Zagreb motorway in Croatia. Furthermore, effects of an opening in the lower portion of the wind barrier wall were investigated and results for bridges without the wind barrier in place were reported as well. Some preliminary results were previously presented in Kozmar et al. (2009b).

\section{Wind tunnel experiments}

Experiments were carried out in the CRIACIV boundary layer wind tunnel described in detail in Augusti et al. (1995) following standard wind-tunnel procedures (Simiu and Scanlan, 1996). This wind tunnel was designed as an open-return (Eiffel) suction-type wind tunnel with a closed test section. Wind velocity through the test section can be regulated between $0 \mathrm{~m} / \mathrm{s}$ and $35 \mathrm{~m} / \mathrm{s}$ by both adjusting the pitch blade angle and regulating the speed of the fan powered by a $160 \mathrm{~kW}$ engine, where the fan is placed at the outlet of the test section. The total length of the wind-tunnel test section (from the nozzle outlet to diffuser inlet) is approximately $22 \mathrm{~m}$. The test section is $1.6 \mathrm{~m}$ high and $2.2 \mathrm{~m}$ wide at the outlet of the nozzle, i.e. at the inlet to the test section. Due to diverging side walls in the longitudinal direction to avoid pressure gradients, the test section width at the center of the turntable is $2.4 \mathrm{~m}$. In some wind-tunnel studies it is required to reproduce the atmospheric boundary layer (ABL) flow. In the CRIACIV boundary layer wind tunnel, the ABL simulation can be generated along the first $11 \mathrm{~m}$ of the test section ( $8 \mathrm{~m}$ long fetch and $3 \mathrm{~m}$ at the turntable, where horizontal incidence angle of the flow can be varied). In this study, the full-scale height of the bridge together with the wind barrier is approximately $7 \mathrm{~m}$ and it can be assumed that differences in flow characteristics of the undisturbed flow do not change significantly with height within these $7 \mathrm{~m}$. Thus, the flow was taken to be uniform and the ABL velocity profile was not reproduced.

2D Particle Image Velocimetry (PIV) measurements were taken on two wind-tunnel bridge models (Hreljin and Bukovo) in the wake of a wind barrier to study the effects of horizontal and vertical angles of attack on mean velocity and vorticity flow field characteristics. A Dantec 2100 PIV system was employed together with two Quantel Big Sky lasers (power output $220 \mathrm{~mJ}$ per light impulse), a CCD Kodak Megaplus camera with Nikon optics (60 mm, resolution $1 \mathrm{MPx}$ and frequency filter), a Le Maitre smoke generator, and a Dantec PIV processor with Flowmap 3.61 software for data measurements and analysis. In this study, the time interval between two light impulses within one frame was $60 \mu \mathrm{s}$ and the total number of frames for one configuration was 200 . The final result is a velocity and vorticity map calculated as an average out of 200 frames. Vorticity is the measure which has been chosen to evaluate the turbulence structure of the flow behind the barrier; due to the possibility of high variations of the flow velocity, vorticity is more suitable to show the intensity and the structure of the turbulence with respect to other possible mapping of the flow (e.g. rms values). Simultaneously with PIV measurements, velocities in the undisturbed flow well upwind from the bridge models were taken by using a Dantec single hotwire and a Prandtl-Pitot tube.

Wind-tunnel models of the Hreljin and Bukovo viaducts were made out of wood for the length scale factor 1:66 and they are presented in full-scale dimensions in Fig. 1 with a wind barrier already in place. The simulation length scale was chosen to satisfy two contradictory demands, i.e. blockage of the test section and the critical Reynolds number. In particular, wind-tunnel models were manufactured as large as possible to obtain larger Reynolds numbers. However, increasing the model size inevitably increases the blockage of the wind-tunnel test section; in wind-tunnel tests a maximum tolerable blockage between $5 \%$ and $6 \%$ should not be exceeded (e.g. Simiu and Scanlan, 1996). The freestream velocity in all tests was kept at approximately $12 \mathrm{~m} / \mathrm{s}$, as bridge model vibrations were observed at larger velocities. Reynolds number calculated using the height of the model bridge section without the barrier $(7.5 \mathrm{~cm})$ and average freestream velocity $(12 \mathrm{~m} / \mathrm{s})$ was approximately $6 \times 10^{4}$. In all tests, the blockage was less than $6 \%$, including structural models and measuring equipment placed in the wind-tunnel test section, indicating the air flow around structural models and their aerodynamic behavior in the wind tunnel is a good representation of prototype conditions, as suggested in Hucho (2002) and Holmes (2001). The Reynolds 
a

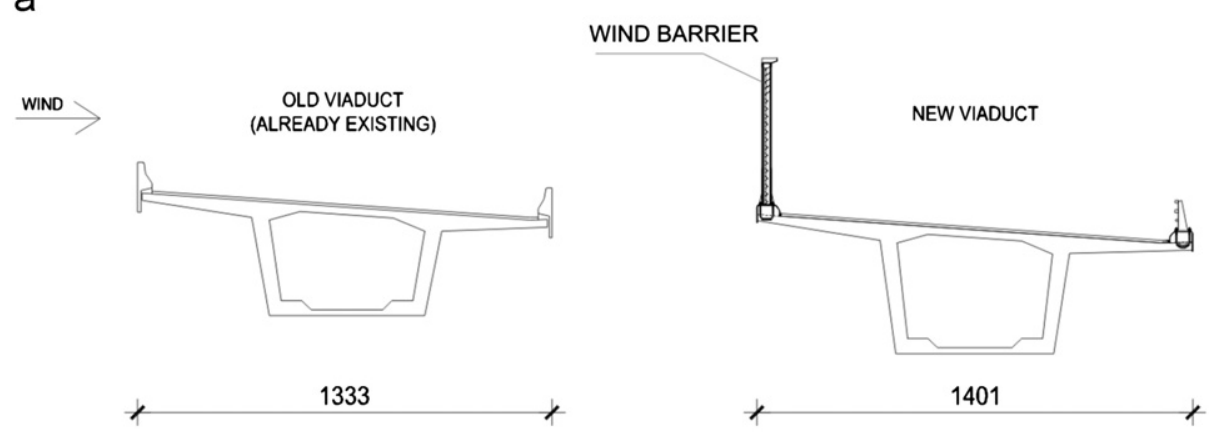

b

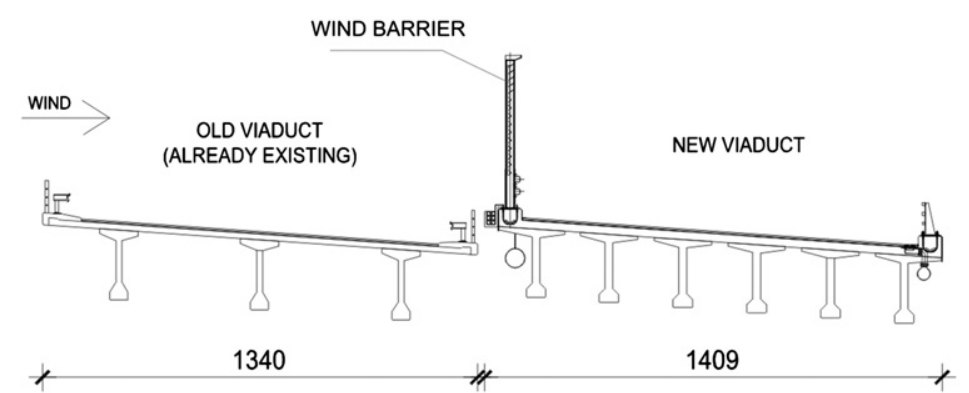

Fig. 1. Viaducts: (a) Hreljin and (b) Bukovo (quotes in $\mathrm{cm}$, full-scale), as reported in Procino et al. (2008).

number sensitivity of the aerodynamics of bluff bodies with sharp edges was studied by Larose and D'Auteuil (2006), who indicated that it is hard at a first glance to define a general minimum Reynolds number above which these effects will be limited. In their review, Larose and D'Auteuil (2006) reported critical Reynolds numbers for several bridges. Schewe (2001) argues that most global Reynolds number effects on bluff bodies can be attributed to fundamental variations in the topological structure of the wake with variations in values of Reynolds number. Furthermore, it was suspected that the location of the laminarto-turbulent transition originates from these structural changes.

Bridge section model width (normal to main wind direction) to bridge span ratio was approximately $1: 3$ to avoid possible effects of bridge model side supports on flow field characteristics. PIV velocity measurements were carried out in the vertical plane in the middle of central wind barrier segment, between two pillars carrying the wind barrier, to avoid flow disturbances caused by the vertical structural elements. In the full-scale, every segment of the wind barrier consists of two pillars at $2.5 \mathrm{~m}$ distance and the porous wall between them. These $2.5 \mathrm{~m}$ wide wind barrier segments were supposed to be placed on the Hreljin and Bukovo viaducts detached from each other, to avoid transferring of vibrations along the viaducts.

Both at Hreljin and Bukovo sites, the doubling of the existing bridges was designed. As the characteristics of existing (old) bridges for the Hreljin and Bukovo viaducts do not allow placing of wind barriers, only the protection of vehicles on new bridges was studied. Hence, the flow field characteristics were investigated for new bridges only, which were to be constructed, as presented on the right-hand side in Fig. 1(a) and (b). As the wind is expected to blow mainly from left to right, as reported in Fig. 1, in periods of strong cross-winds it is planned to redirect vehicles from old bridge onto the new one. The traffic on the new bridge would be two-way instead of one-way until cross-winds become weaker resulting in improved safety conditions for vehicles on the old bridge. In general, the purpose of this study was to propose a wind barrier design which would improve the safety and comfort of passengers on new bridges by reducing the windinduced instability of vehicles. Special attention was paid to flow characteristics between the road surface and $4 \mathrm{~m}$ height, as standard tractor-trailer height is $4 \mathrm{~m}$ (Wong, 2001) and this type of vehicle proved to be the most vulnerable to cross-wind effects. Valuable sources of information on the performance of high-sided vehicles on roads and bridges exposed to cross winds were extensive studies by Baker (1991a,b,c).

For both the Hreljin and Bukovo viaducts, barriers are constituted by horizontal $2.1 \mathrm{~mm}$ high elements with a ' $\mathrm{V}$ ' shape, placed $2.1 \mathrm{~mm}$ apart in vertical direction, both dimensions given at model scale. When considering pillars carrying the wind barrier as a part of the wall, too, it gives a porosity equal to $42 \%$ (calculated as the open area between ' $V$ ' profiles compared to the entire area of the wall); effects of the barrier wall porosity on flow field characteristics were reported in more detail in Procino et al. (2008). For the Hreljin viaduct, barrier height is $7.5 \mathrm{~cm}$ model-scale $(5 \mathrm{~m}$ full-scale when scaled-up using the scale factor 1:66) and it is manufactured using ' $\mathrm{V}$ ' shaped elements, oriented with the spire toward the wind $(\rightarrow<)$. In the Bukovo tests, barrier height is $6 \mathrm{~cm}$ model-scale ( $4 \mathrm{~m}$ full-scale when scaled-up using the scale factor 1:66) and barrier elements are oriented against the wind $(\rightarrow>)$. Both orientations of wind barrier elements create very similar flow field characteristics behind the barrier, as reported in Procino et al. (2008); hence, it is expected they would perform similarly in terms of sheltering efficiency for vehicles. In addition, it is worth to be mentioned that two lowest ' $\mathrm{V}$ ' profile elements in the Bukovo barrier wall were not in place, i.e. there was a $0.7 \mathrm{~cm}$ and $1.1 \mathrm{~cm}$ modelscale high opening ( $0.46 \mathrm{~m}$ and $0.73 \mathrm{~m}$ full-scale, respectively) in the lower part of the barrier. The idea was to investigate flow field characteristics for a case without a lower part of wind barrier. In this paper, effects of wind incidence angle on flow field characteristics for the Hreljin and Bukovo viaducts were reported for low-turbulent $\left(I_{u}=3 \%\right)$ and high-turbulent $\left(I_{u}=17 \%\right)$ wind. Turbulence intensity $I_{u}$ in both cases was calculated based on hot-wire measurements in the undisturbed flow. The incoming wind turbulence was generated by using wooden grids positioned at the inlet of the wind-tunnel test section, as presented in Fig. 2. The turbulent flow was found to be reasonably uniform across the model position, which was $10 \mathrm{~m}$ downstream of the grid. Effects of variations in horizontal incidence angle $\alpha$ were studied for the Hreljin viaduct for $\alpha=0^{\circ}$ and $\alpha=15^{\circ}$, as 
a

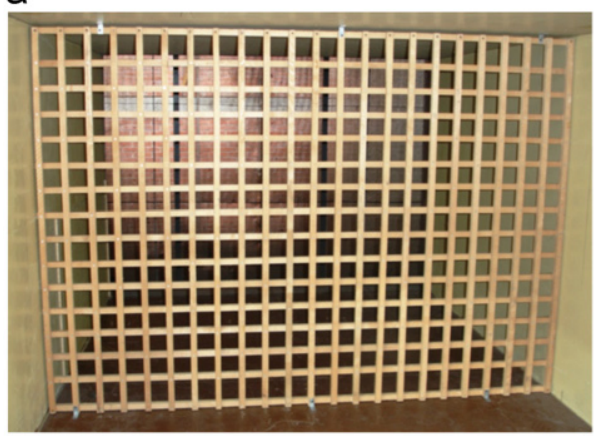

b

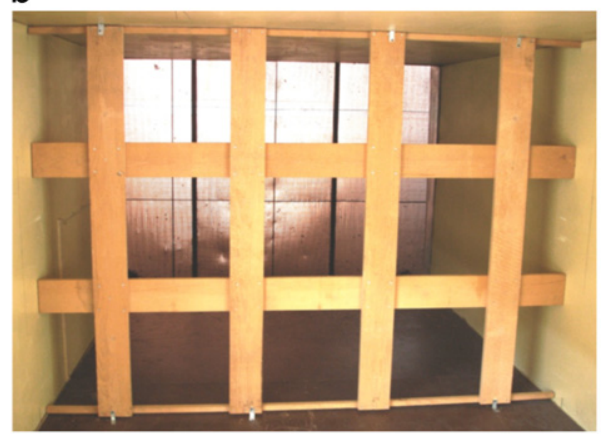

Fig. 2. Turbulence generating grid at the inlet of the wind-tunnel test section: (a) $I_{u}=3 \%$ and (b) $I_{u}=17 \%$.

a

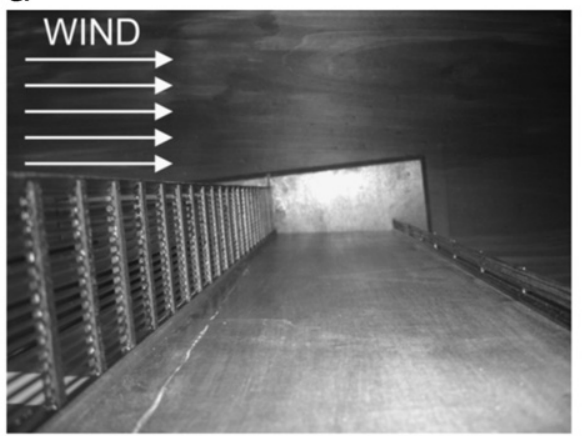

b

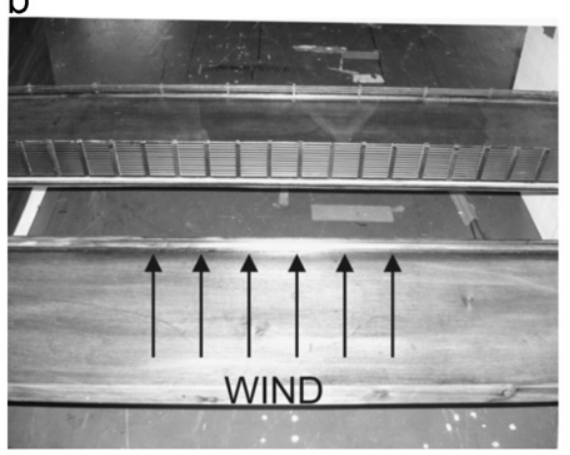

C



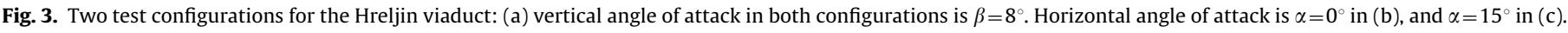

presented in Fig. 3. Vertical angle of attack $\beta$ for Hreljin was $8^{\circ}$ in both configurations, as that was supposed to be a vertical wind incidence angle in respect to the terrain once the bridge has been constructed. Effects of the vertical wind incidence angle $\beta$ were tested on the Bukovo model for $\beta=0^{\circ}, \beta=5^{\circ}$ and $\beta=10^{\circ}$, while the horizontal angle of attack $\alpha$ was $0^{\circ}$ in all the Bukovo tests, as presented in Fig. 4. The wind incidence angles are defined in respect to the line normal to the wind barrier. Due to design reasons, both the Hreljin and Bukovo bridges have a slope of $4^{\circ}$ toward the trailing edge of the bridge, as it can be seen in Fig. 1. Bridge section models were placed in the center of the wind-tunnel cross-section to avoid possible boundary effects of wind-tunnel side walls on air flow characteristics. The Hreljin viaduct illuminated with a PIV laser beam is shown in Fig. 5.

\section{Results and discussion}

In this section, 2D mean velocity fields on bridge sections behind wind barriers, measured using the PIV, are reported as normalized with the freestream velocity $\bar{u}_{\infty}$, which was measured by using a Pitot tube. In addition, 2D vorticity fields are presented as well. In the following, turbulence intensity has been evaluated only in the undisturbed flow upwind from the bridge, by using measurements performed by a hot-wire; hot-wire measurements were not taken on the bridge section model as previous studies (see Coleman and Baker, 1992) reported doubts on the accuracy of the hot-wire velocity and turbulence intensity measurements behind wind barriers due to the recirculating nature of the flow field. In diagrams, height $H$ and length $L$ are normalized by using the wind barrier height $B(7.5 \mathrm{~cm}$ for Hreljin viaduct and $6 \mathrm{~cm}$ for Bukovo viaduct at model scale). For a convenient discussion, $H$ and $L$ are addressed in full-scale dimensions as well. Preliminary tests were taken for bridges without a barrier and the obtained results are reported in Fig. 6 .

Without a wind barrier, wind velocities on bridge models reach $80 \%$ of the freestream velocity at height as low as $H / B$ ranges from 0.3 to 0.4 , that is around $1 \mathrm{~m}$ full-scale, along with very strong vorticity in the immediate vicinity of the road surface. This trend was observed for both the Hreljin and Bukovo viaducts. In general, the airflows around vehicles exposed to cross-winds are dominated by the alternate shedding of vortices from the top 
a

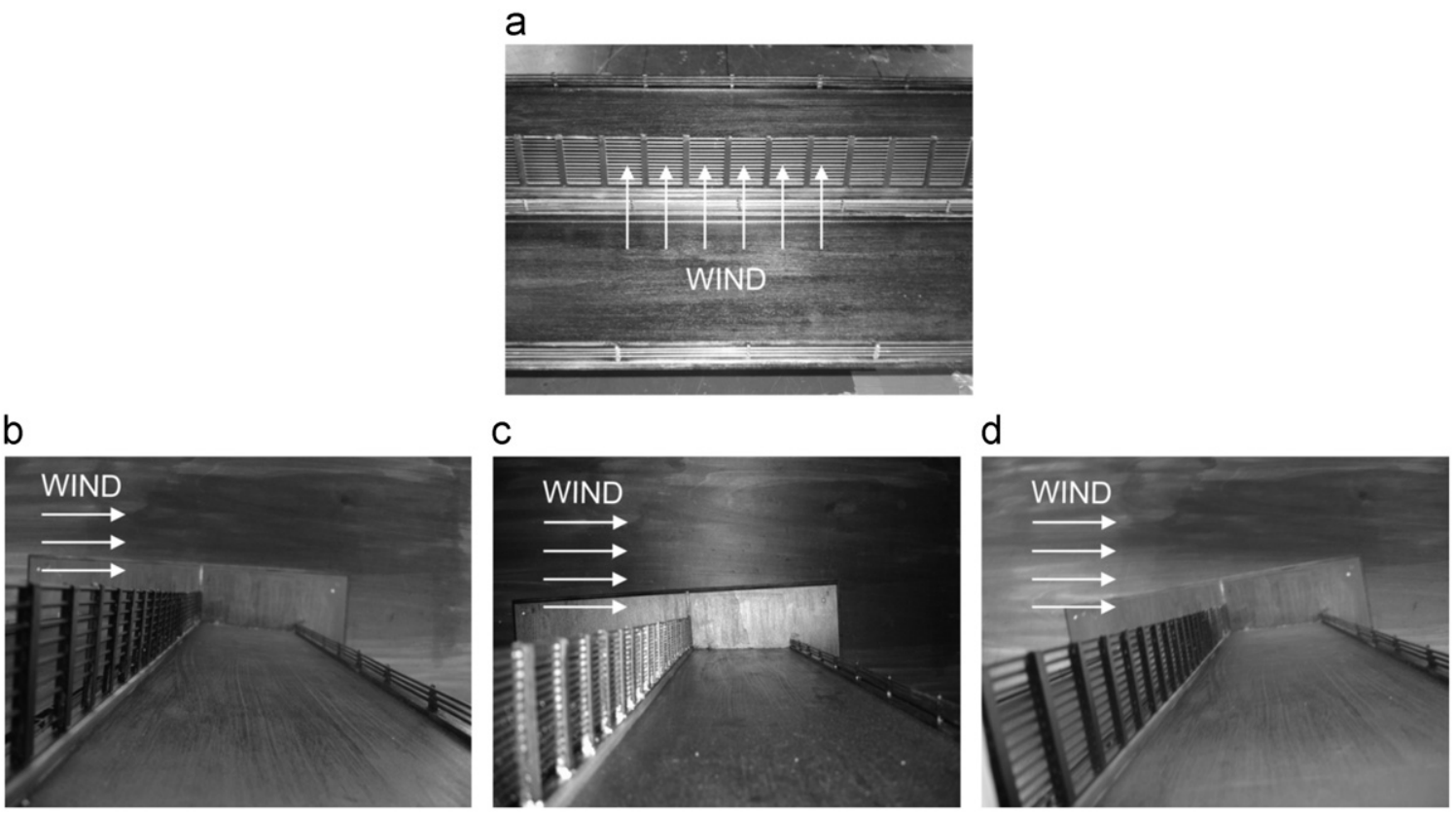

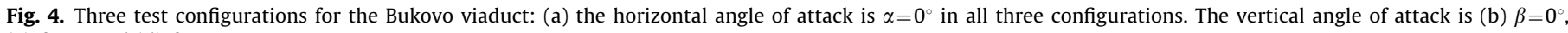
(c) $\beta=5^{\circ}$ and (d) $\beta=10^{\circ}$.

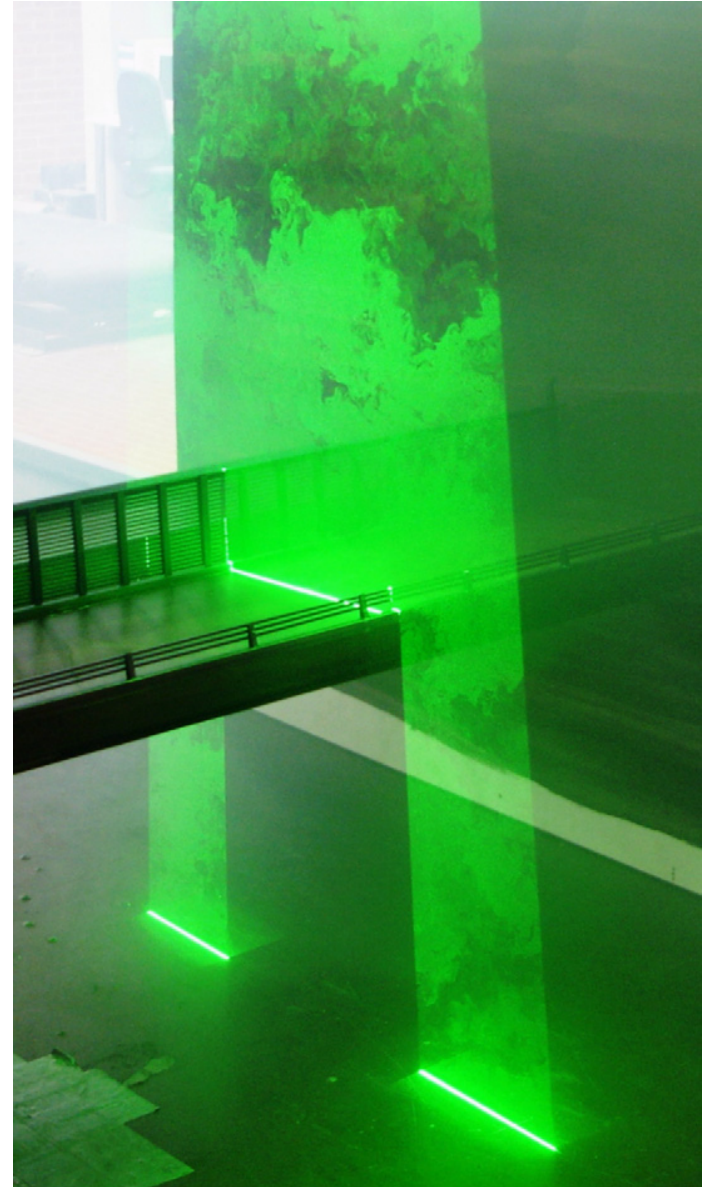

Fig. 5. Wind-tunnel model of the Hreljin viaduct illuminated with a PIV laser beam.

and bottom of vehicles (Coleman and Baker, 1992). Hence, it is expected that vehicles on the bridge without wind barriers, as reported in Fig. 6, would experience strong instability due to high average velocity, as well as due to strong vorticity close to surface. In particular, it is expected that the strong suction within the separated flow immediately behind the leading edges of the vehicle and an intensified flow separation for this configuration would significantly deteriorate vehicle maneuverability in comparison with configurations with wind barriers in place, as Coleman and Baker (1994) indicate that the vortex occurring at the leading edge of the vehicle and flow separation are the dominant flow mechanisms for high-sided vehicles in cross winds. It is of interest that, whilst the vortex shedding from the vehicle surface clearly determines the magnitude and frequency of the lift force fluctuations, it does not seem to affect side force fluctuations to any great extent (Coleman and Baker, 1994). On the other hand, the unsteady side force experienced by vehicles on bridges without a barrier is predominantly due to turbulence buffeting (e.g. Cooper, 1984) and vortex shedding from the leading edge of the bridge (Kozmar et al., 2009c, 2011). Therefore, it is expected that a wind barrier would significantly alter flow conditions and reduce wind loading of vehicles behind the barrier.

Effects of variations in the horizontal incidence angle $\alpha$ on mean velocity field and vorticity field were studied for the Hreljin viaduct and reported for low-turbulent wind $\left(I_{u}=3 \%\right)$ in Fig. 7 , and for high-turbulent wind $\left(I_{u}=17 \%\right)$ in Fig. 8.

Flow field characteristics dependent on the vertical angle of attack $\beta=0^{\circ}, 5^{\circ}$ and $10^{\circ}$ were presented for the Bukovo viaduct for low-turbulent wind $\left(I_{u}=3 \%\right)$ in Fig. 9, and for high-turbulent wind $\left(I_{u}=17 \%\right)$ in Fig. 10. It needs to be mentioned that disturbances of the flow near the barrier walls were observed for both the Hreljin and Bukovo viaducts due to difficulties with the measuring technique. Consequently, values recorded in this region were omitted from diagrams.

In general, wind velocities downstream from the wind barrier are significantly lower compared to the freestream velocity $\bar{u}_{\infty}$ upwind from the barrier, in agreement with Coleman and Baker (1992). In this study, behind the barrier mean wind velocities reach $0.6 \bar{u}_{\infty}$ at maximum that represents a $40 \%$ reduction compared to the undisturbed flow. The only exception is the small region immediately behind the barrier at the Bukovo viaduct 
a

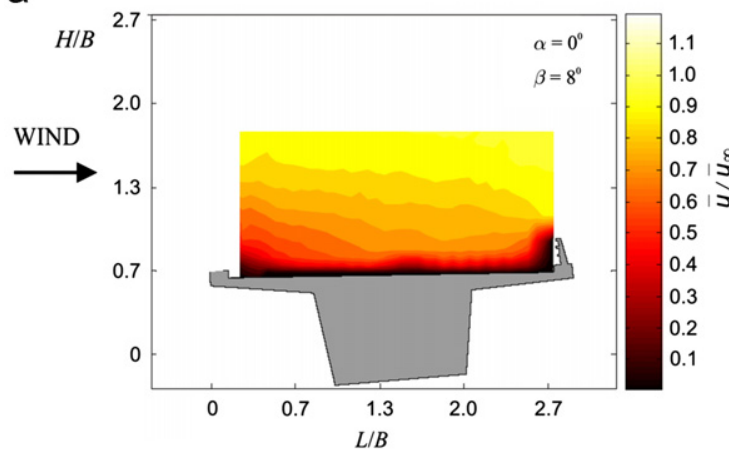

C

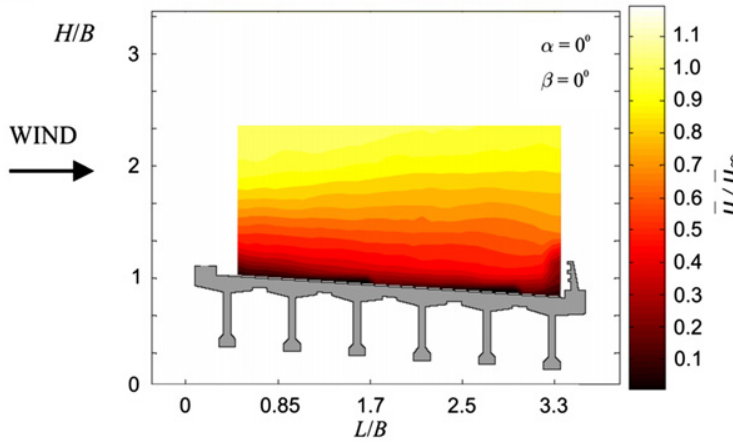

b

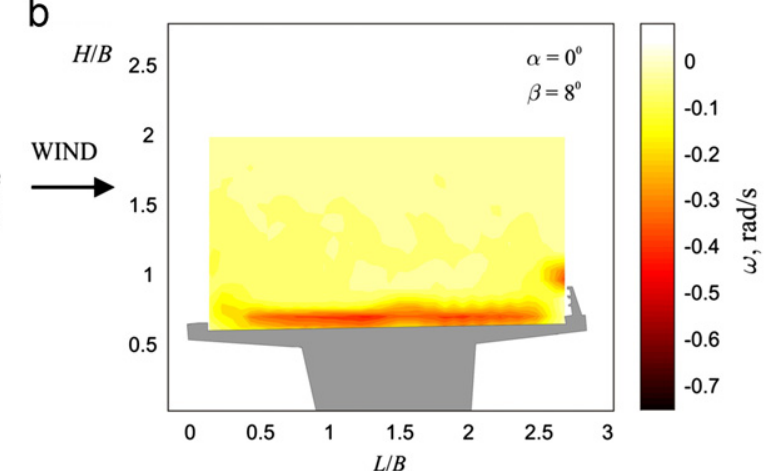

d

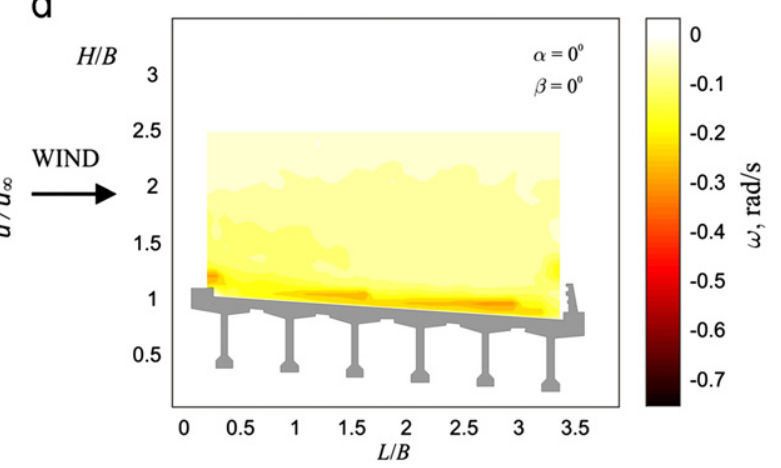

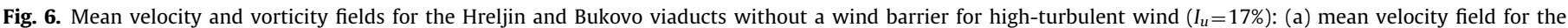

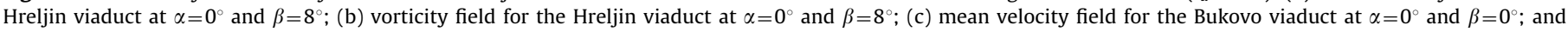
(d) vorticity field for the Bukovo viaduct at $\alpha=0^{\circ}$ and $\beta=0^{\circ}$.

a

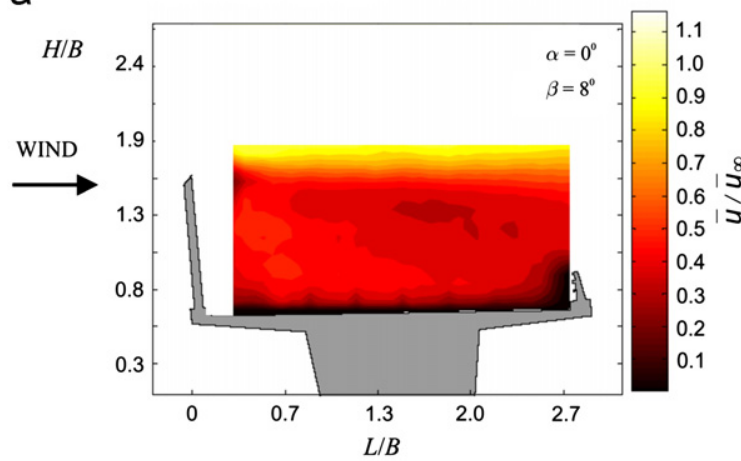

C

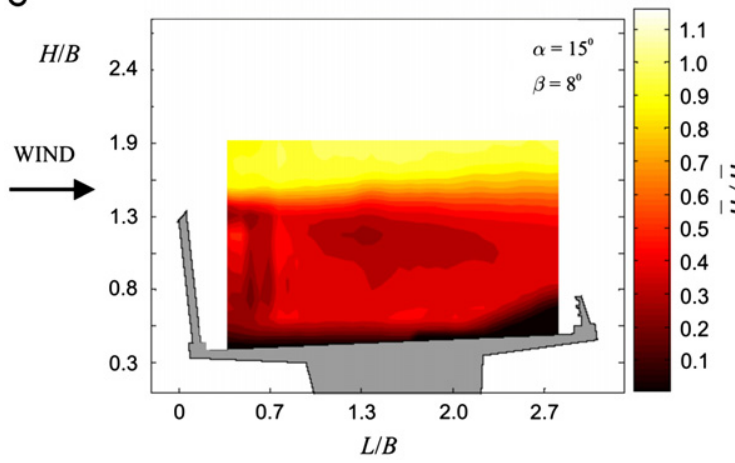

b

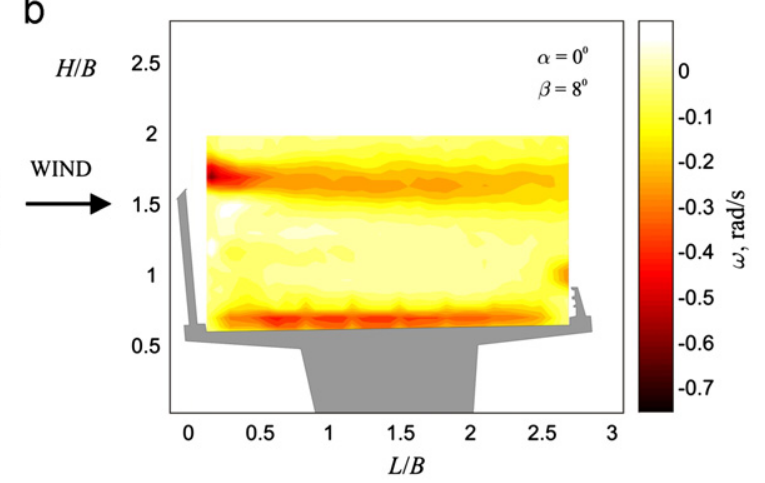

d

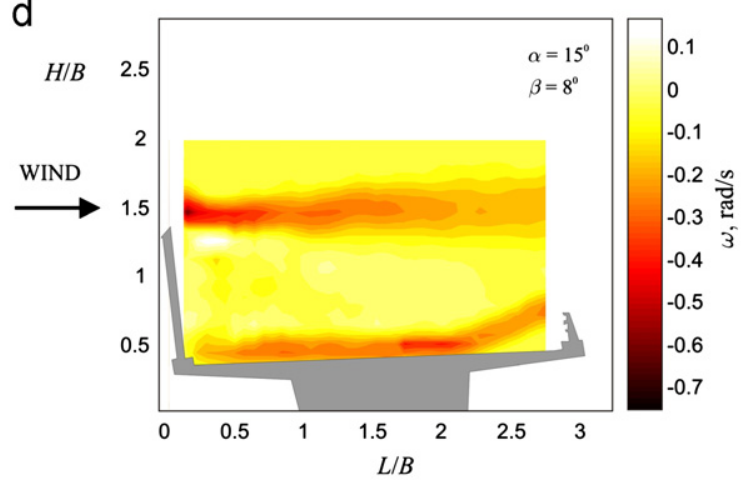


$\beta=8^{\circ}$, (c) mean velocity field for $\alpha=15^{\circ}$ and $\beta=8^{\circ}$ and (d) vorticity field for $\alpha=15^{\circ}$ and $\beta=8^{\circ}$.

where mean velocities around $0.9 \bar{u}_{\infty}$ were observed due to an opening in the lower part of the barrier wall, as presented in Figs. 9-11. Mean velocities reach their maximum immediately behind the barrier and decrease further downstream. Strong velocity fluctuations were observed downstream from the top of the wind barrier and immediately above the road surface due to 
a

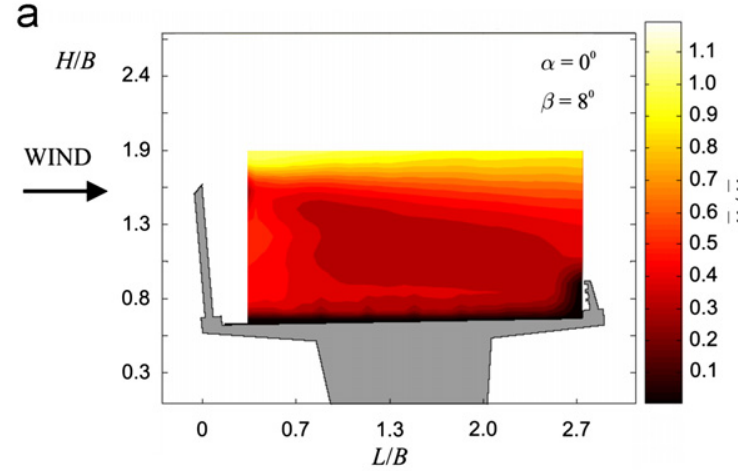

C

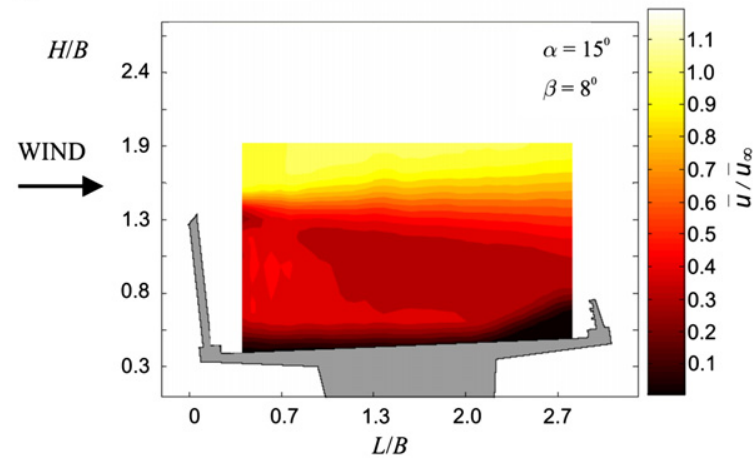

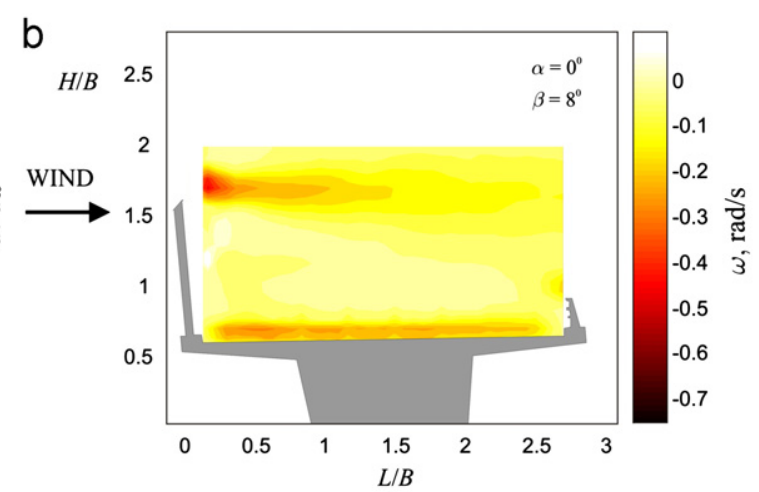

d

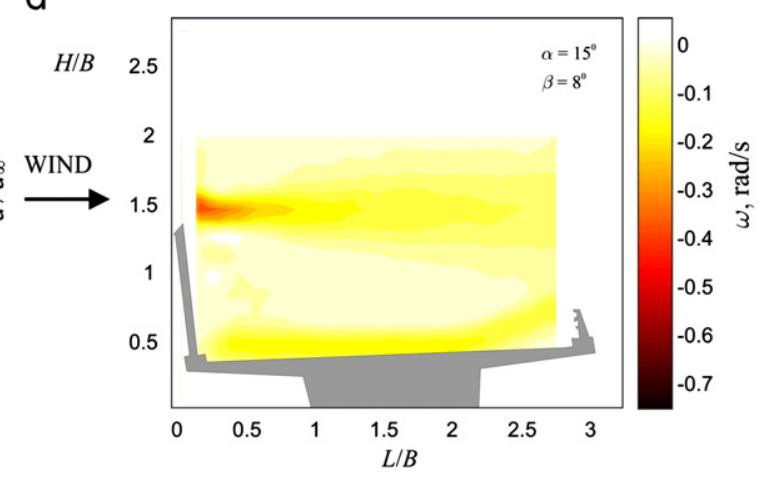


and $\beta=8^{\circ}$, (c) mean velocity field for $\alpha=15^{\circ}$ and $\beta=8^{\circ}$ and (d) vorticity field for $\alpha=15^{\circ}$ and $\beta=8^{\circ}$.

flow separation from the wind barrier top and from the leading edge corners of the bridge, respectively. In general, the separated flow region is divided from the outer flow by a thin region of high shear and vorticity, a region known as the free shear layer, which is similar to the boundary layer on an airfoil, but not attached to a surface. In addition, Coleman and Baker (1992) report a vastly increased turbulence intensity produced by the wakes of wind barrier elements along with the characteristic vortex shedding from the barrier that was reported in Figs. 7 and 8 as well.

For the Hreljin viaduct, areas with low mean velocities and intensified vorticity were observed close to road surface that is in part due to the shedding of vortices from the leading edge of the bridge. Similar phenomenon was observed for the Bukovo viaduct as well. When observing the effects of the horizontal incidence angle $\alpha$ on flow field characteristics, similar trends were reported both for low-turbulent wind $\left(I_{u}=3 \%\right)$ and for high-turbulent $\left(I_{u}=17 \%\right)$ wind. Variations in the horizontal wind incidence angle $\alpha$ do not seem to affect the flow field characteristics significantly, as shown in Figs. 7 and 8 . Mean velocities for both $\alpha=0^{\circ}$ and $\alpha=15^{\circ}$ reach $0.6 \bar{u}_{\infty}$ at maximum and vorticity $\omega$ is around $0.1 \mathrm{rad} / \mathrm{s}$ through the majority of the measuring domain, while larger $\omega$ around $0.2 \mathrm{rad} / \mathrm{s}$ were observed only close to road surface. The freestream vorticity was observed to be close to zero.

Due to similar flow field characteristics behind wind barriers for different horizontal incidence angles it is expected that the vehicles would perform dynamically in a similar way, independently from wind horizontal direction. In addition, introducing wind barriers that in turn reduce average wind velocity and shift strong vorticity away from the road surface would have several benefits for the vehicle stability. In particular, it could be expected that a substantial reduction of the side and lift forces acting on the vehicle would be obtained in comparison with a configurations without wind barriers in place, in agreement with Coleman and Baker (1992). In addition, it is expected that the vehicle maneuverability would be significantly improved as well, as the wheels would not be exposed to the strong transient dynamic loading that was the case in configuration without wind barriers, where the vorticity close to surface was reported to be much stronger. Also, the unsteady lift force controlled by the unsteady shedding of vortices from the top and bottom of the vehicle would be expected to be weaker for the configuration with wind barriers in place, as vortex shedding is expected to be weaker at reduced averaged velocities and vorticity behind the barriers.

Downslope wind effects on vehicles were simulated by changing the vertical angle of attack $\beta$ on the wind-tunnel model of the Bukovo viaduct. Results in Figs. 9 and 10 indicate an increase in mean velocities behind the wind barrier with increasing vertical incidence angle $\beta$. Therefore, strong velocity fluctuations due to flow separation from the wind barrier top and mean freestream velocities approach closer to the road with increasing vertical incidence angle $\beta$. This phenomenon could possibly induce wind-induced instability of vehicles, especially in highsided trucks in the traffic lane close to trailing edge of the bridge. In particular, it is expected that large velocity and vorticity approaching the road surface in the area close to trailing edge of the bridge at larger vertical wind incidence angles would increase unsteady side and lift forces due to an intensified vortex shedding from vehicles. Hence, it is expected that the vehicles would become more vulnerable to cross-wind effects at larger vertical wind incidence angles. In the Bukovo tests, strong vorticity appears due to flow separation from the barrier top at $H / B$ is 1 ( $4 \mathrm{~m}$ full-scale) and close to the road due to vortex shedding from the leading edge of the bridge. In the traffic lane immediately behind the wind barrier, there is a region of large mean velocity and a strong vorticity close to road surface. It is due to an opening in the wind barrier close to road surface on the Bukovo viaduct (lowest two barrier elements were removed from the barrier wall), which allows strong winds to pass through the 
a

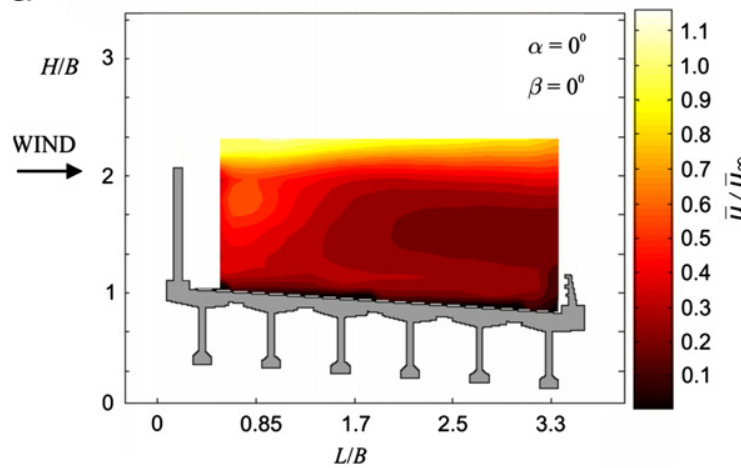

C

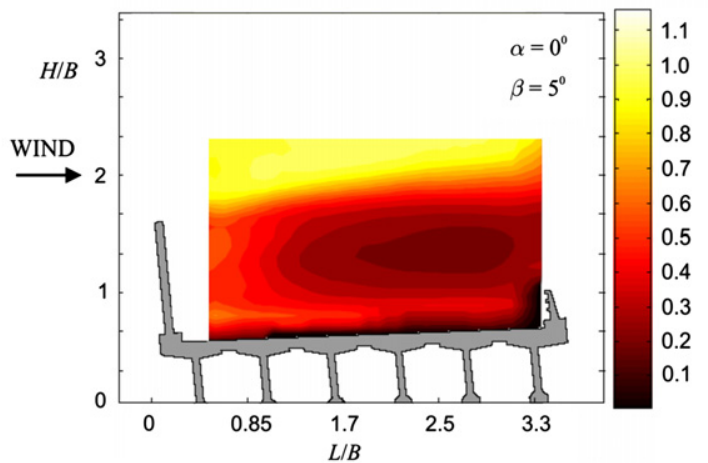

e



b

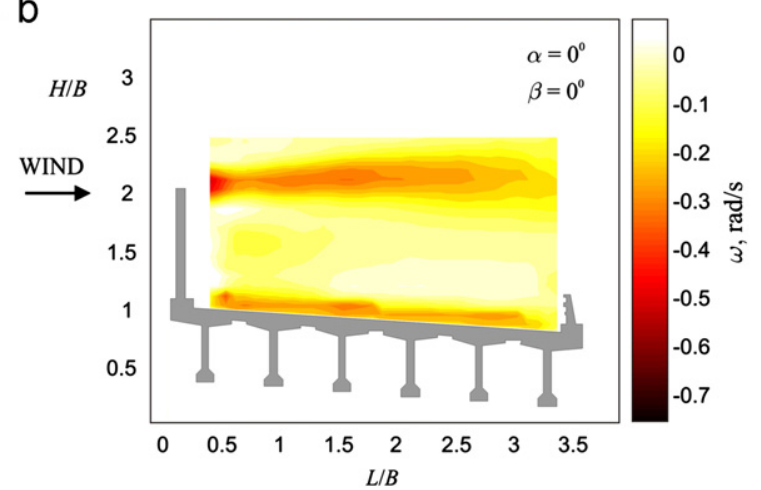

d

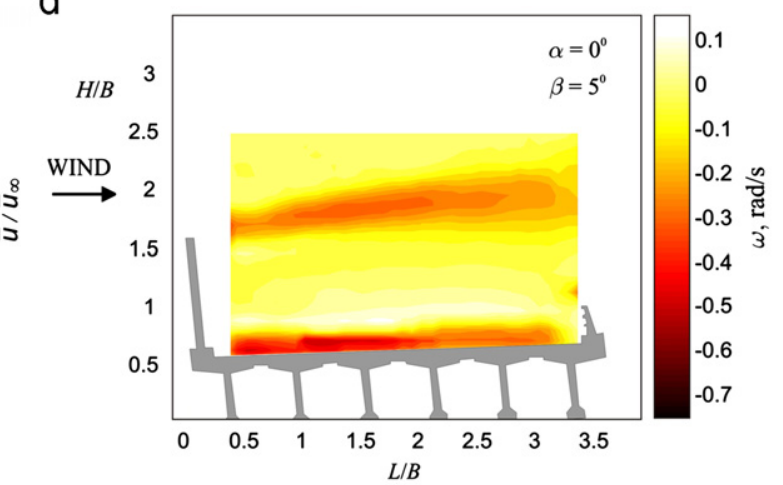

f

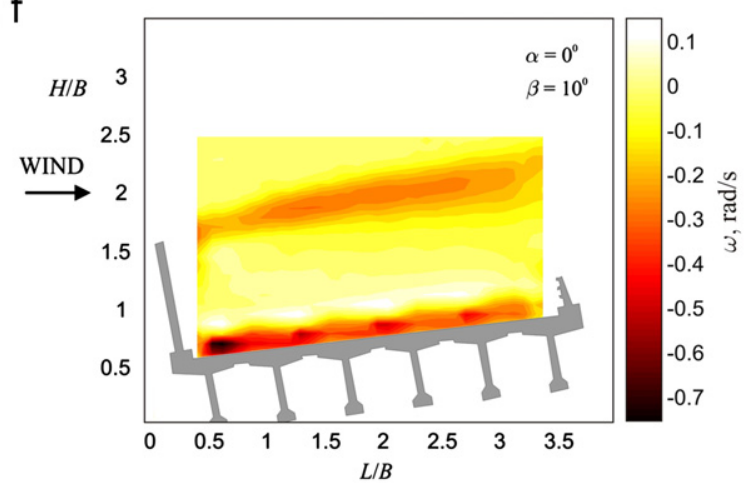

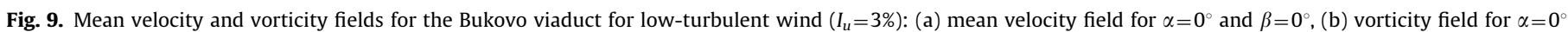

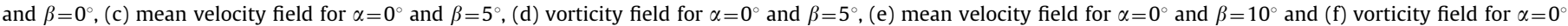
and $\beta=10^{\circ}$.

barrier without significant loss of momentum. These effects become stronger with an increased vertical incidence angle $\beta$, i.e. for $\beta=10^{\circ}$ velocities equal to freestream velocity $\bar{u}_{\infty}$ and vorticity around $0.7 \mathrm{rad} / \mathrm{s}$ were observed in this localized area. A significant decrease in wind barrier sheltering efficiency due to an opening in the wind barrier wall, as observed in this study, agrees very well with results previously reported by Klingbeil et al. (1982). In general, effects of the vertical wind incidence angle $\beta$ on the flow field characteristics were similar for lowturbulent wind $\left(I_{u}=3 \%\right)$ and for high-turbulent $\left(I_{u}=17 \%\right)$ wind.

Additional tests were carried out for the Bukovo viaduct to further investigate effects of an opening in the wind barrier wall on flow field characteristics. Mean velocity and vorticity fields reported in Fig. 11 were recorded in three different configurations, i.e. one test with a full barrier wall without an opening, and two tests with $0.46 \mathrm{~m}$ and $0.73 \mathrm{~m}$ high openings (full-scale dimensions), i.e. $H / B$ is 0.115 and 0.183 , respectively. In all tests reported in Fig. 11, horizontal incidence angle $(\alpha)$ is $0^{\circ}$, vertical incidence angle $(\beta)$ is $10^{\circ}$, turbulence intensity $\left(I_{u}\right)$ is $17 \%$, and the porosity of the barrier wall is $42 \%$.

Experimental results for mean velocity fields clearly indicate a development of small, localized area with very high velocities nearly equal to freestream velocity with an increased height of an opening in the lower portion of a barrier wall. This area is observed at $L / B$ is 0.25 ( $1 \mathrm{~m}$ full-scale) downstream of the wind barrier. This phenomenon is expected to give rise to the side and lift forces experienced by vehicles and to decrease their dynamic performances. Simultaneously, the vorticity above the road surface becomes stronger in the entire protected area behind the wind barrier. In the region close to surface, strong and localized vortices show clear periodicity, as they were recorded at a space interval equal to $L / B=0.65$ to 0.70 (i.e. every 2.6 to $2.8 \mathrm{~m}$ full-scale) along the transversal direction of the bridge. This phenomenon is due to periodic vortex shedding from the top of the barrier and the flow passing through an opening in the barrier wall. It is expected that periodic, localized regions of strong vorticity predominantly characterized by the bridge architecture 
a

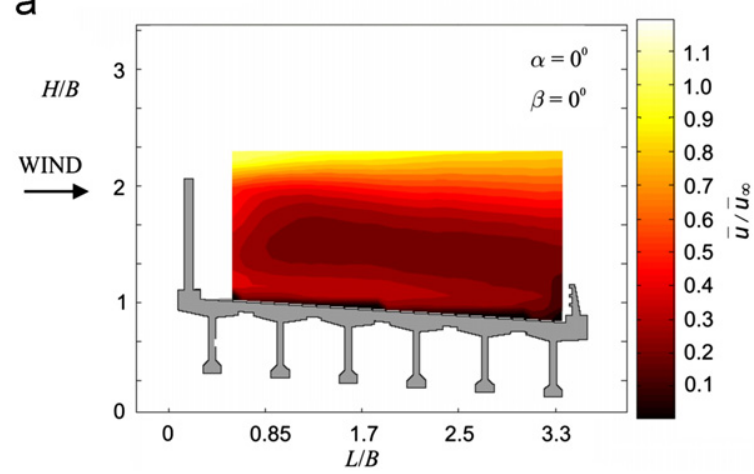

C

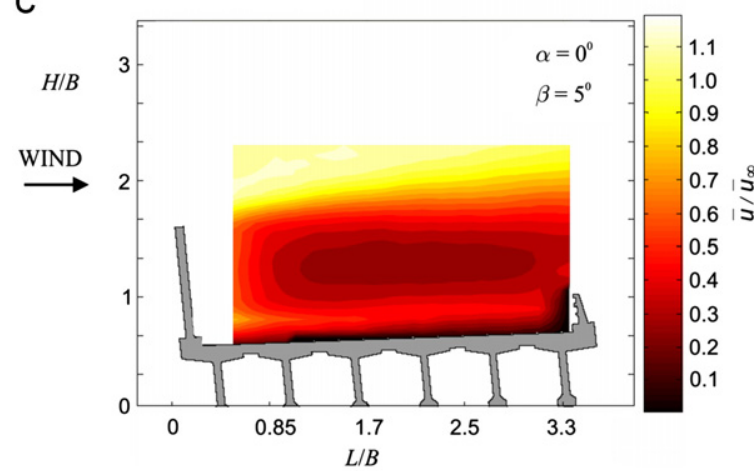

e

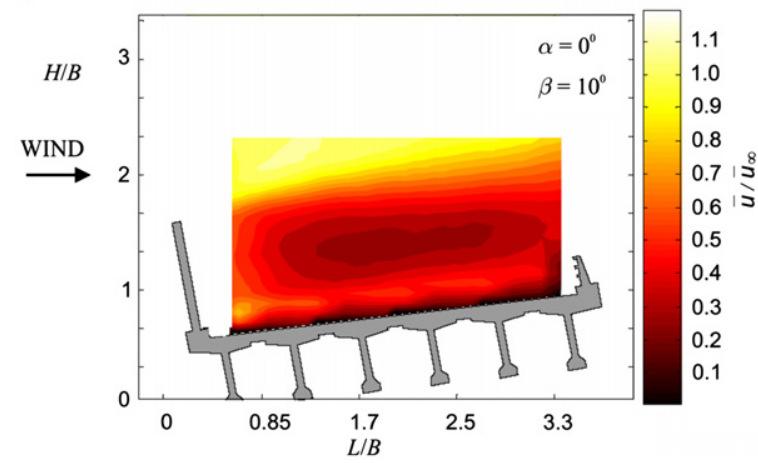

b

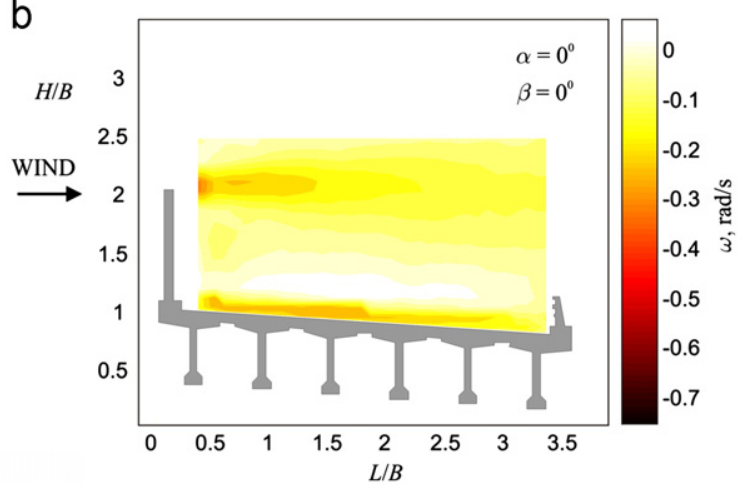

d
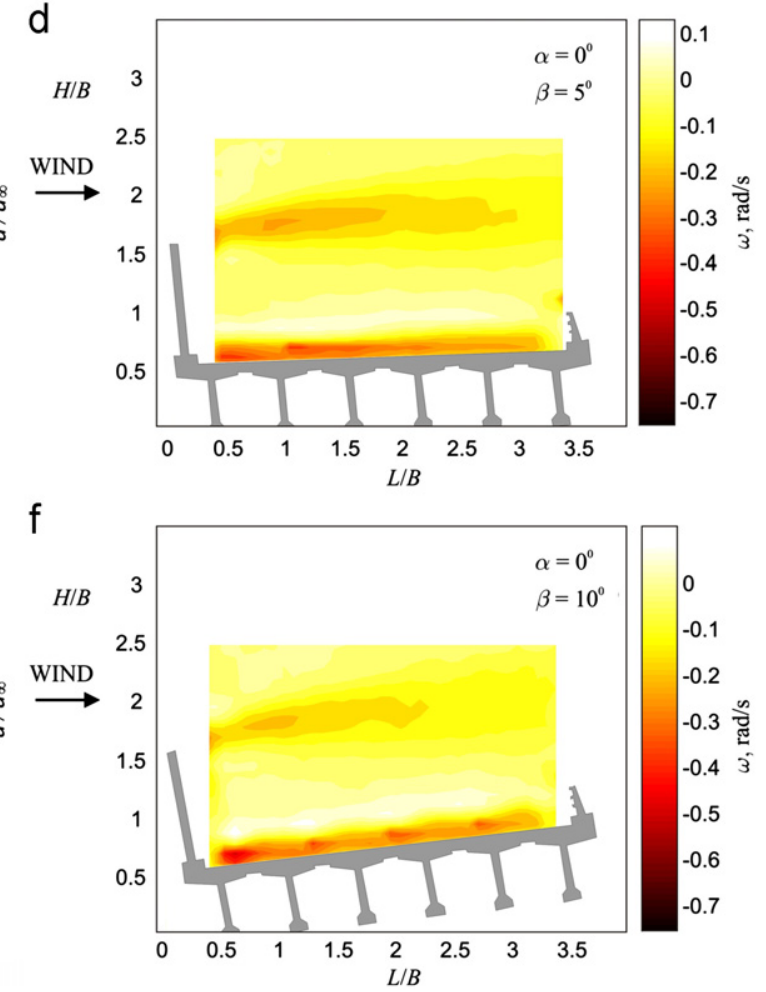

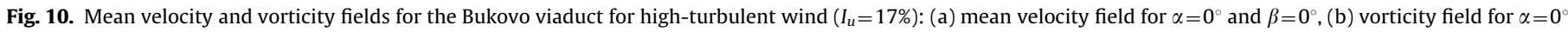

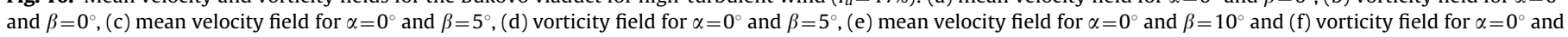
$\beta=10^{\circ}$.

and an opening in the barrier wall would significantly alter a resonant interaction with vehicle suspension dynamics. Hence, reduced vehicle stability can be expected for this case. In addition, it would be difficult to make a parallel between the vortex shedding frequency characteristic for bridges St $\sim 0.1$ (e.g. Ryall et al., 2000) and the observed periodicity reported in Fig. 11, as in the present study the flow across the bridge with the wind barrier in place was already significantly changed by crossing the upwind bridge, flowing through/above the wind barrier, through an opening in the barrier wall. In general, missing wind barrier elements in the lower portion of the barrier wall result in larger velocities and stronger vorticity that may increase wind-induced forces and moments experienced by vehicles on bridges.

\section{Concluding remarks}

Wind-tunnel tests were carried out to evaluate effects of wind incidence angle on flow field characteristics in the wake of wind barriers on bridges. Experimental results indicate a possibility of wind-induced instability of high-sided vehicles at larger vertical incidence angles, especially in the traffic lane close to trailing edge of the bridge, as velocity fluctuations and mean freestream velocities approach the road surface when increasing vertical wind incidence angle. Removing elements from the wind barrier enhances large local velocities immediately downstream from the barrier wall and stronger vorticity in the entire area behind the wind barrier wall. Variations in horizontal incidence angle do not seem to affect flow field characteristics significantly. Without a wind barrier, mean wind velocities on bridge reach $80 \%$ of the freestream velocity at height as low as $1 \mathrm{~m}$ full-scale along with very strong vorticity in the immediate vicinity of the road surface. In general, wind barrier induces lower mean velocity and higher turbulence on the bridge. The reduction in mean velocity is expected to cause a lower horizontal wind drag on trucks, while increased turbulence alters the vortex-shedding mechanism around trucks, resulting in a lower vertical force. 
a

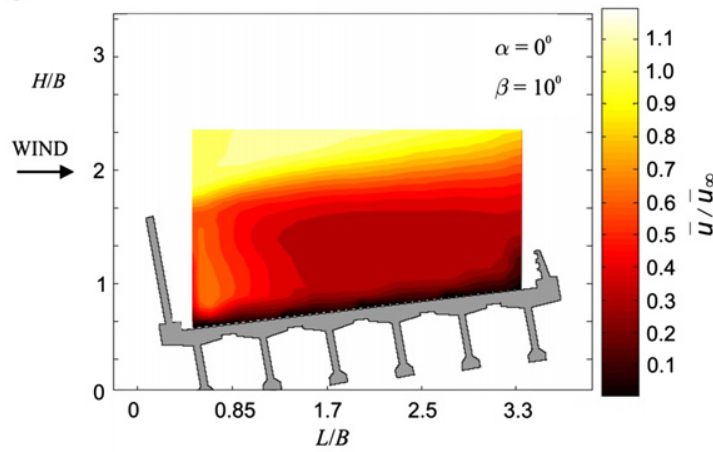

C

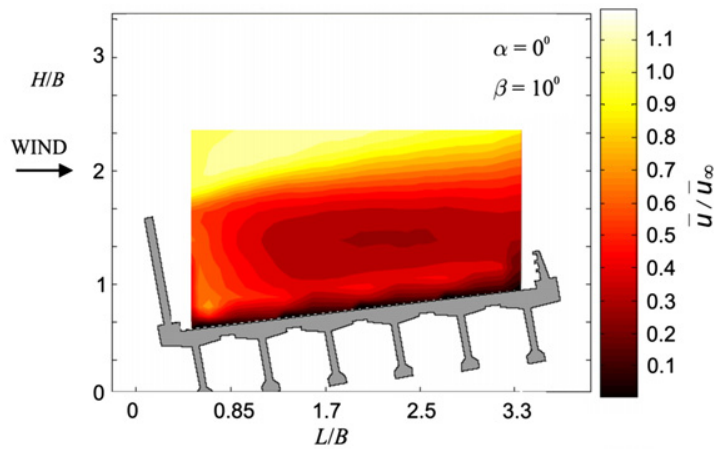

e

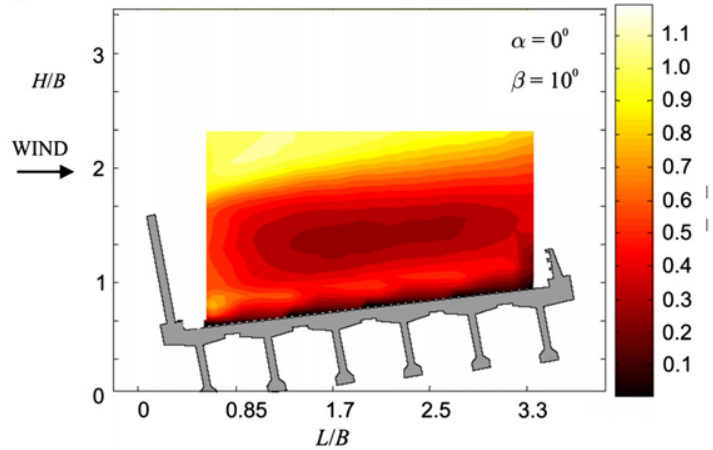

b

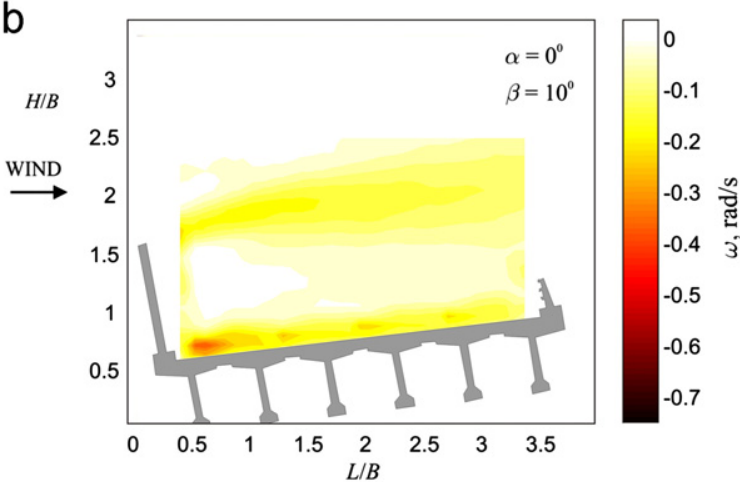

d

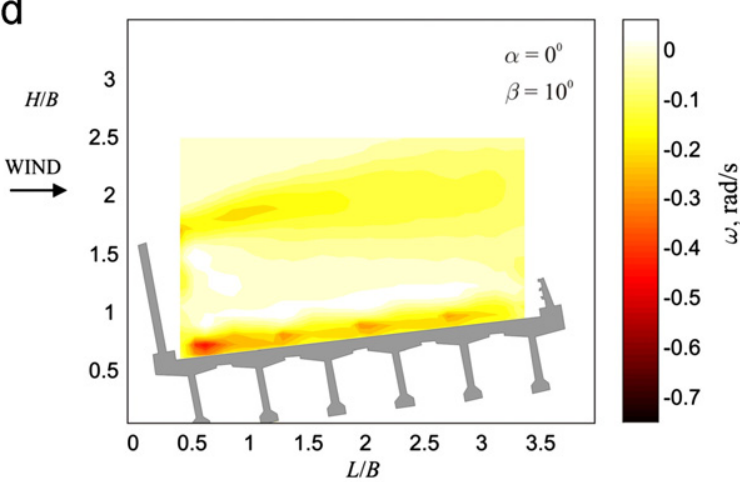

f

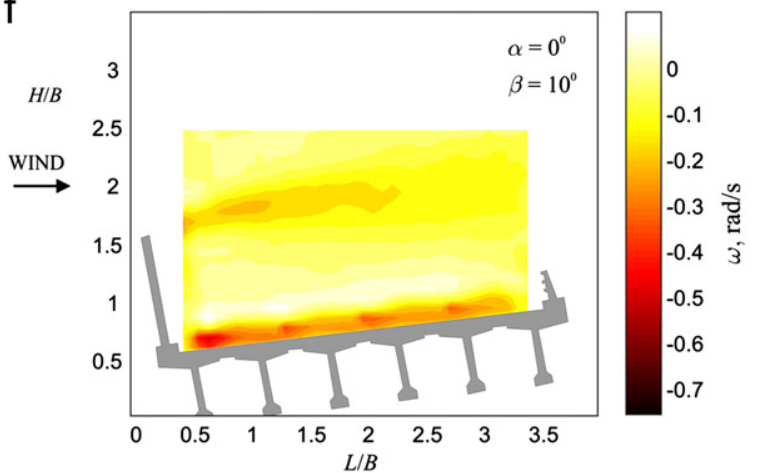

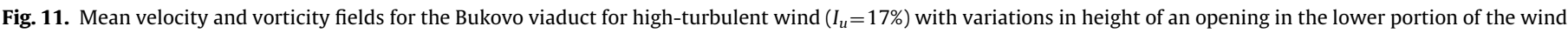

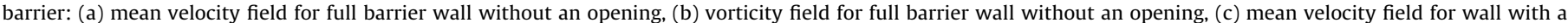

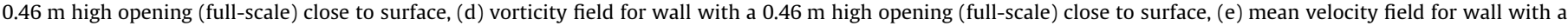
$0.73 \mathrm{~m}$ high opening (full-scale) close to surface and (f) vorticity field for wall with a $0.73 \mathrm{~m}$ high opening (full-scale) close to surface.

\section{Acknowledgments}

This study was carried out in collaboration among the InterUniversity Research Center on Wind Engineering and Building Aerodynamics (CRIACIV), Italy, the Faculty of Mechanical Engineering and Naval Architecture (FSB), University of Zagreb, Croatia, and the Inženjersko Projektni Zavod (IPZ) of Zagreb, Croatia. The authors acknowledge helpful discussions with Mr. Zoran Lončarec (IPZ) and Prof. Zdravko Virag (FSB).

\section{References}

Arie, M., Rouse, H., 1956. Experiments on two-dimensional flow over a normal wall. Journal of Fluid Mechanics 1, 129-141.

Augusti, G., Spinelli, P., Borri, C., Bartoli, G., Giachi, M., Giordano, S., 1995. The C.R.I.A.C.I.V. atmospheric boundary layer wind tunnel. In: Proceedings of the 9th International Conference on Wind Engineering (ICWE), New Delhi, India.

Baker, C.J., 1991a. Ground vehicles in high cross winds. 1. Steady aerodynamic forces. Journal of Fluids and Structures 5, 69-90.
Baker, C.J., 1991b. Ground vehicles in high cross winds. 2. Unsteady aerodynamic forces. Journal of Fluids and Structures 5 (1), 91-111.

Baker, C.J., 1991c. Ground vehicles in high cross winds. 3. The interaction of aerodynamic forces and the vehicle system. Journal of Fluids and Structures 5 (2), 221-241.

Blenk, H., Trienes, H., 1956. Strömungstechnische Beiträge zum Windschutz: Strömungsuntersuchungen an Windhindernissen am Modell und in der freien Natur, Grundlagen der Landtechnik. VDI Verlag, Düsseldorf (in German).

Coleman, S.A., Baker, C.J., 1992. The reduction of accident risk for high sided road vehicles in cross winds. Journal of Wind Engineering and Industrial Aerodynamics 41-44, 2685-2695.

Coleman, S.A., Baker, C.J., 1994. An experimental study of the aerodynamic behaviour of high sided lorries in cross winds. Journal of Wind Engineering and Industrial Aerodynamics 53, 401-429.

Cooper, R.K., 1984. Atmospheric turbulence with respect to moving ground vehicles. Journal of Wind Engineering and Industrial Aerodynamics 17 (2), 215-238.

Cornelis, W.M., Gabriels, D., 2005. Optimal windbreak design for wind-erosion control. Journal of Arid Environments 61, 315-332.

de Bray, B.G., 1971. Protection by fences. In: Proceedings of the Seminar on Wind Effects on Buildings and Structures, University of Auckland.

Dierickx, W., Gabriels, D., Cornelis, W.M., 2001. Wind tunnel study on wind speed reduction through successive synthetic windscreens. Journal of Agricultural Engineering Research 79 (1), 117-123. 
Dierickx, W., Cornelis, W.M., Gabriels, D., 2003. Wind tunnel study on rough and smooth surface turbulent approach flow and on inclined windscreens. Biosystems Engineering 86 (2), 151-166.

Dong, Z., Luo, W., Qian, G., Wang, H., 2007. A wind tunnel simulation of the mean velocity fields behind upright porous fences. Agricultural and Forest Meteorology 146, 82-93.

Frank, C., Ruck, B., 2005. Double-arranged mound-mounted shelterbelts: influence of porosity on wind reduction between the shelters. Environmental Fluid Mechanics 5, 267-292.

Gandemer, J., 1979. Wind shelters. Journal of Industrial Aerodynamics 4, 371-389.

Gandemer, J., 1981. The aerodynamic characteristics of windbreaks, resulting in empirical design rules. Journal of Wind Engineering and Industrial Aerodynamics 7, 15-36.

Good, M.C., Joubert, P.N., 1968. The form drag of two-dimensional bluff plates immersed in turbulent boundary layers. Journal of Fluid Mechanics 31 (3), 547-582.

Heisler, G.M., DeWalle, D.R., 1988. Effects of windbreak structure on wind flow. Agriculture, Ecosystems \& Environment 22/23, 41-69.

Holmes, J.D., 2001. Wind loading of structures. Spon Press, London.

Hucho, W.-H., 2002. Aerodynamik der stumpfen Körper. Vieweg \& Sohn, Braunschweig/Wiesbaden (in German).

Jensen, M., 1954. Shelter Effect: Investigations into the Aerodynamics of Shelter and Its Effects on Climate and Crops. The Danish Technical Press, Copenhagen.

Jensen, M., 1958. The model law for phenomena in the natural wind. Ingenioren 2 , 121-128.

Judd, M.J., Raupach, M.R., Finnigan, J.J., 1996. A wind tunnel study of turbulent flow around single and multiple windbreaks, Part I: velocity fields. Boundary-Layer Meteorology 80, 127-165.

Kaiser, H., 1959. Die Strömung an Windschutzstreifen. Berichte Des Deutschen Wetterdienstes 53 (7), 1-36 (in German).

Klingbeil, K., Benndorf, D., Grunert, F., 1982. Aerodynamische Grundlagen für Windschutzpflanzungen: Der Einfluss der geometrischen Struktur von Gehölzschutzstreifen auf ihre Schutzwirkung. Meteorologische Zeitschrift 32 (3), 165-175. (in German).

Kozmar, H., Procino, L., Borsani, A., Bartoli, G., 2009a. Testing aerodynamic properties of road windscreens. Gradevinar 61 (3), 261-267. (in Croatian).

Kozmar, H., Procino, L., Bartoli, G., Borsani, A., 2009b. Wind barriers on bridges: effects of wind incidence angle on flow field characteristics. In: Proceedings of the 7th Asia-Pacific Conference on Wind Engineering, Taipei, Taiwan.

Kozmar, H., Butler, K., Kareem, A., 2009c. Aerodynamic loads on a vehicle exposed to cross-wind gusts: an experimental study. In: Proceedings of the 7th AsiaPacific Conference on Wind Engineering, Taipei, Taiwan.

Kozmar, H., Butler, K., Kareem, A., 2011. Effects of the cross-wind gust strength on vehicle aerodynamics. In: Proceedings of the 13th International Conference on Wind Engineering., Amsterdam, The Netherlands.
Larose, G.L., D'Auteuil, A., 2006. On the Reynolds number sensitivity of the aerodynamics of bluff bodies with sharp edges. Journal of Wind Engineering and Industrial Aerodynamics 94, 365-376.

McAneney, K.J., Judd, M.J., 1991. Multiple windbreaks: an aeolian ensemble. Boundary-Layer Meteorology 54, 129-146.

McNaughton, K.G., 1988. Effects of windbreaks on turbulent transport and microclimate. Agriculture, Ecosystems \& Environment 22/23, 17-39.

Miller, D.R., Rosenberg, N.J., Bagley, W.T., 1975. Wind reduction by a highly permeable tree shelterbelt. Agricultural Meteorology 14, 321-333.

Nägeli, W., 1941. Über die Bedeutung von Windschutzstreifen zum Schutze landwirtschaftlicher Kulturen. Schweiz. Z. Forstwiss 92 (11), 265-280 (in German)

Nord, M., 1991. Shelter effects of vegetation belts-results of field measurements Boundary-Layer Meteorology 54, 363-385.

Plate, E.J., 1971. The aerodynamics of shelter belts. Agricultural Meteorology 8, 203-222.

Procino, L., Kozmar, H., Bartoli, G., Borsani, A., 2008. Wind barriers on bridges: the effect of wall porosity. In: Proceedings of the 6th Colloquium on Bluff Bodies Aerodynamics and Applications, Milan, Italy.

Raine, J.K., 1974. Wind protection by model fences in a simulated atmospheric boundary layer. In: Proceedings of the 5th Australasian Conference on Hydraulics and Fluid Mechanics, Christchurch, New Zealand, 200-210.

Raine, J.K., Stevenson, D.C., 1977. Wind protection by model fences in a simulated atmospheric boundary layer. Journal of Wind Engineering \& Industrial Aerodynamics 2 (2), 159-180.

Ryall, M.J., Parke, G.A.R., Harding, J.E., 2000. The Manual of Bridge Engineering. Thomas Telford.

Schewe, G., 2001. Reynolds number effects in flow around a more-or-less bluff bodies. Journal of Wind Engineering and Industrial Aerodynamics 89, 1267-1289.

Schwartz, R.C., Fryrear, D.W., Harris, B.L., Bilbro, J.D., Juo, A.S.R., 1995. Mean flow and shear stress distributions as influenced by vegetative windbreak structure. Agricultural and Forest Meteorology 75, 1-22.

Simiu, E., Scanlan, R.H., 1996. Wind Effects on Structures, $3^{\text {rd }}$ ed. John Wiley \& Sons.

Štrukelj, A., Ciglarič, I., Pipenbaher, M., 2005. Analysis of a Bridge Structure and its Wind Barrier under Wind Loads. Structural Engineering International 15 (4) 220-227.

Wang, D.L., Chen, A.R., Zhou, Z.Y., 2007. Aerodynamic characters of a trans-oceanic cable-stayed bridge with wind barrier, In: Proceedings of the 12th International Conference on Wind Engineering (ICWE), Cairns, Australia, 807-814.

Wong, J.Y., 2001. Theory of Ground Vehicles. John Wiley \& Sons, New York. 